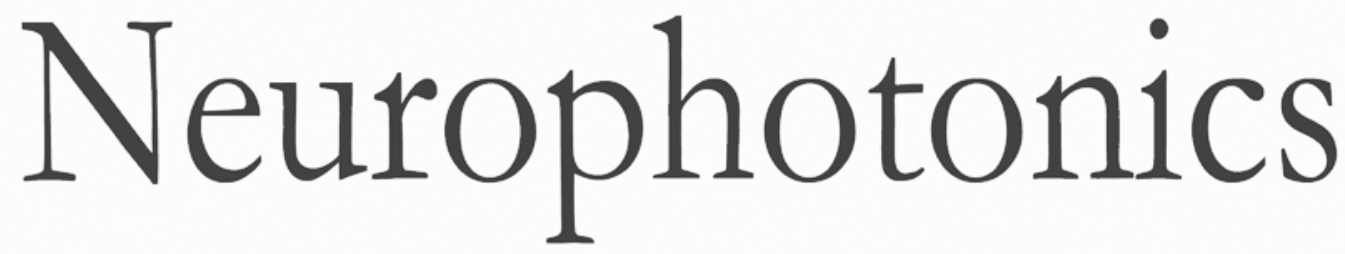

\title{
Interstitial imaging with multiple diffusive reflectance spectroscopy projections for in vivo blood vessels detection during brain needle biopsy procedures
}

Fabien Picot

Andréanne Goyette

Sami Obaid

Joannie Desroches

Simon Lessard

Marie-André Tremblay

Mathias Strupler

Brian Wilson

Kevin Petrecca

Gilles Soulez

Frédéric Leblond 


\title{
Interstitial imaging with multiple diffusive reflectance spectroscopy projections for in vivo blood vessels detection during brain needle biopsy procedures
}

\author{
Fabien Picot, ${ }^{\mathrm{a}, \mathrm{b}}$ Andréanne Goyette,${ }^{\mathrm{a}, \mathrm{b}}$ Sami Obaid, ${ }^{\mathrm{b}}$ Joannie Desroches, ${ }^{\mathrm{a}, \mathrm{b}}$ Simon Lessard, ${ }^{\mathrm{b}}$ \\ Marie-André Tremblay, ${ }^{\mathrm{a}, \mathrm{b}}$ Mathias Strupler, ${ }^{\mathrm{a}}$ Brian Wilson, ${ }^{\mathrm{c}}$ Kevin Petrecca, ${ }^{\mathrm{d}}$ Gilles Soulez, ${ }^{\mathrm{b}}$ and \\ Frédéric Leblond ${ }^{a, b, *}$ \\ apolytechnique Montreal, Department of Engineering Physics, Montreal, Québec, Canada \\ ${ }^{\mathrm{b}}$ Centre de Recherche du Centre Hospitalier de I'Université de Montréal, Montreal, Québec, Canada \\ 'University Health Network/University of Toronto, TMDT 15-314, Toronto, Ontario, Canada \\ dMcGill University, Brain Tumour Research Center Montreal Neurological Institute and Hospital, \\ Department of Neurology and Neurosurgery, Montreal, Québec, Canada
}

\begin{abstract}
Blood vessel injury during image-guided brain biopsy poses a risk of hemorrhage. Approaches that reduce this risk may minimize related patient morbidity. We present here an intraoperative imaging device that has the potential to detect the brain vasculature in situ. The device uses multiple diffuse reflectance spectra acquired in an outward-viewing geometry to detect intravascular hemoglobin, enabling the construction of an optical image in the vicinity of the biopsy needle revealing the proximity to blood vessels. This optical detection system seamlessly integrates into a commercial biopsy system without disrupting the neurosurgical clinical workflow. Using diffusive brain tissue phantoms, we show that this device can detect 0.5-mm diameter absorptive carbon rods up to $\sim 2 \mathrm{~mm}$ from the biopsy window. We also demonstrate feasibility and practicality of the technique in a clinical environment to detect brain vasculature in an in vivo model system. In situ brain vascular detection may add a layer of safety to image-guided biopsies and minimize patient morbidity. ๑ The Authors. Published by SPIE under a Creative Commons Attribution 4.0 Unported License. Distribution or reproduction of this work in whole or in part requires full attribution of the original publication, including its DOI. [DOI: 10.1117/1.NPh.6.2.025003]
\end{abstract}

Keywords: optics; surgery; biopsy; light transport; tomography; spectroscopy; tissue optics; diffuse reflectance.

Paper 18069R received Oct. 29, 2018; accepted for publication Feb. 20, 2019; published online Apr. 23, 2019.

\section{Introduction}

The standard of care for malignant brain tumors includes opencranium surgery ${ }^{1-8}$ to achieve maximal resection of cancer tissue followed by adjuvant therapies such as chemotherapy and radiotherapy. ${ }^{1-8}$ However, surgery is not always the optimal treatment choice because the risks associated with this procedure can outweigh potential benefits. This aspect is especially important for patients with poor health or when lesions are difficult to access either because they are deeply seated or they are associated with multiple focal sites. ${ }^{1-8}$ In these cases a brain needle biopsy can be performed to assess the potential benefits of a surgical resection procedure by providing clinicians with a more precise diagnosis. During the biopsy procedure, bleeding can occur in $0.3 \%$ to $59.8 \%$ of cases. ${ }^{9-14}$ Although most of these instances of tissue damage are asymptomatic or can lead to minor transient effects (e.g., dizziness and headaches), more serious consequences can result from brain biopsy procedures because of damage incurred to larger and more critical blood vessels. For example, there is a small yet non-negligible mortality risk (up to $3.9 \%$ in some studies) associated with this procedure. $9,15,16,17$

Several optical imaging techniques are currently being developed for intraoperative blood vessel detection. Methods have been developed using the fluorescence from indocyanine green (ICG) to help guide procedures in the ocular fundus, in laparoscopic surgery, in stereotactic brain surgery, in robot-

*Address all correspondence to Frédéric Leblond, E-mail: frederic.leblond@ polymtl.ca assisted cardiac procedures, and in esophagectomy. ${ }^{18-22} \mathrm{~A}$ promising method for intraoperative blood vessel detection is optical coherence tomography (OCT), which has been successfully developed for visualization of retinal vasculature and for blood vessel detection in rodent brain. ${ }^{23-25}$ Although OCT cannot interrogate areas larger than a few millimeters across, whereas fluorescence can be used for macroscopic imaging (several centimeters across), the advantages of OCT are its volumetric imaging capabilities (up to 1 to $2 \mathrm{~mm}$ depth sampling mostly depending on tissue absorption) and the fact it does not rely on the administration of an exogenous contrast agent. Fluorescence and OCT are also combined in some studies to enhance the visualization of blood vessels, ${ }^{26-28}$ and more recently an OCT imaging needle has successfully demonstrated its ability to detect blood vessels in human brain surgery. According to that study ${ }^{29}$ OCT can provide high-resolution images in real time and in particular Doppler OCT can provide specific blood vessels detection due to its inherent ability for motion detection. However, the requirement to provide imaging capabilities (e.g., possibly using a mechanical scanning device) makes it difficult to integrate OCT technology into existing commercial biopsy devices, thereby typically requiring an extra step toward the procedural biopsy sample harvesting workflow. Other optical imaging techniques that could be developed for interstitial and intraoperative blood vessel detection include laser Doppler imaging. In this technique, light scattering from moving molecules results in a frequency shift and collecting Doppler-shifted scattered photons interacting with blood vessels provide blood flow information. ${ }^{30,31}$ This method has already been successfully implemented in the human brain. ${ }^{32}$ And 
another study has introduced a new Doppler probe embedded on a needle to fit the requirements for blood vessel detection during brain biopsy procedures. ${ }^{33}$ In a similar way, speckle imaging uses the fluctuation in interference light patterns to reconstruct movement in the scattering medium. This technique has shown that it can detect superficial blood flow in animal and human in vivo studies. ${ }^{34,35}$

In previous work, we have introduced interstitial diffuse optical tomography and have demonstrated its ability to detect highly absorbing inclusions in tissue-simulating diffusing phantoms. ${ }^{36,37}$ The technique was first introduced in Ref. 36, where a proof-of-concept study was presented using a realistic brain tissue phantom to demonstrate that optical tomography can detect several 1-mm diameter high-contrast absorbing objects located $<2 \mathrm{~mm}$ from the edge of a needle. Following our initial work, ${ }^{37}$ we presented a more detailed sensitivity analysis to establish the intrinsic detection limits of the method based on simulations and experiments using brain tissue phantoms. It is demonstrated that absorbers can be detected with diameters $>300 \mu \mathrm{m}$ located up to $>2 \mathrm{~mm}$ from the biopsy needle core for bulk optical properties consistent with brain tissue. The clinical need to improve safety requires the detection of blood vessels within up to $2 \mathrm{~mm}$ from the outer surface of the needle, as this distance is representative of the volume of tissue that is aspirated during tissue extraction. This preliminary work is a proof of principle providing evidence that this new method could be used for the detection of blood vessels (either large vessels or areas containing high densities of small microvessels) during brain needle biopsy procedures in order to minimize the risk of hemorrhage. Here, we present the next step in this research, where a protocol is developed and implemented for in vivo swine brain diffuse optical imaging during a brain biopsy procedure. This animal proof of principle demonstrates that multiple projection diffuse reflectance spectroscopy could be used to guide the insertion of brain biopsy needles by using hemoglobin $(\mathrm{Hb})$ as a surrogate for blood vessels detection prior to sample harvesting. In particular, we present the development of an intraoperative biopsy needle with an integrated multiprojection diffuse optical fiber optics spectroscopy system to detect blood vessels in the vicinity of the biopsy window with the objective of improving procedural safety. We first detail the design and fabrication method for the needle probe that is directly integrated onto a commercial brain biopsy system. An in vitro proof-of-concept study using phantoms made of carbon rods and Intralipid ${ }^{\mathrm{TM}}$ is then presented to demonstrate the feasibility of absorptive inclusions detection in a scattering medium. Finally, we present intraoperative blood vessel detection in vivo during one swine brain biopsy procedure using the diffuse optical imaging system to demonstrate the feasibility and practicality of the technique in a clinical environment. The algorithms for light transport modeling and imaging are also briefly detailed.

\section{Methods}

\subsection{Imaging System}

\subsubsection{Optical brain biopsy needle}

The diffuse reflectance fiber optics system is integrated directly onto the outer cannula of a commercial brain biopsy needle (model 9733068, Medtronic, Colorado), as shown in Fig. 1. The commercial brain biopsy needle is composed of two hollow tubes herein labeled as the inner and the outer cannulas (Fig. 1). The outer cannula is $20 \mathrm{~cm}$ in length with an outer diameter of $2 \mathrm{~mm}$. At the distal end of each cannula, there is a small rectangular opening (henceforth referred to as the biopsy window) of dimensions $1.5 \times 8 \mathrm{~mm}$ through which a sample can be aspirated, cut (through rapid rotation of the inner cannula relative to the outer cannula), and then harvested for pathology analyses. A requirement for the design of the optical biopsy needle is to ensure that the imaging system could be integrated directly on a commercial biopsy needle for minimal disruption of the surgical workflow. The integrated probe consists of 18 optical fibers (NA 0.22 , Low-OH, $105 \mu \mathrm{m}$ core diameter, FG105LCA, Thorlabs) disposed circumferentially around a 200-deg portion of the outer cannula of the commercial brain biopsy needle, leaving a blind spot (Fig. 1). The fibers are disposed opposite the biopsy window, and the angular range they cover is limited in order to leave the later unobstructed. As a result, imaging could be achieved on the opposite side of the window, and hence the need to rotate the probe by 180 deg between optical measurement and tissue collection. The probe design is compatible with
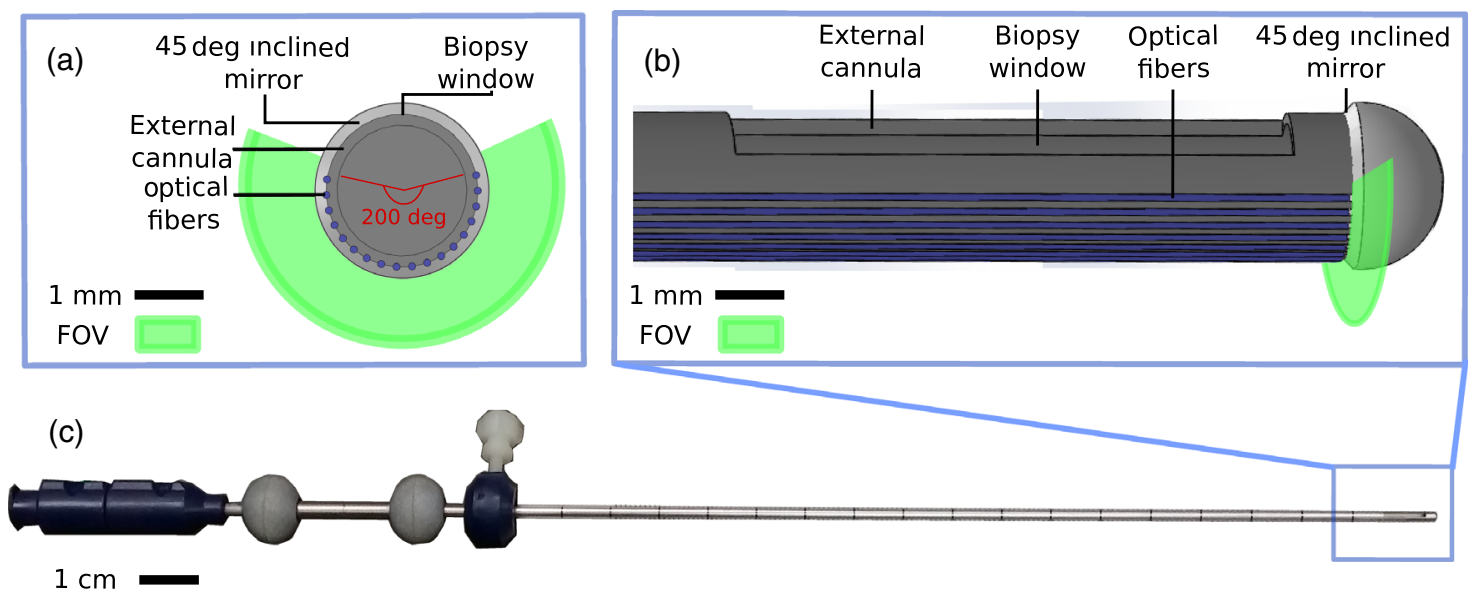

Fig. 1 Optical probe design showing the distribution of the optical fibers around the needle as well as the position of the mirror. Only the external cannula of the commercial needle is shown, with the optical fibers (in blue) disposed on its exterior surface: (a) transverse view and (b) longitudinal view of the optical needle shown in (c). 
the use of a standard stereotactic frame that is used for needle guidance, which allows to precisely control its rotation angle to ensure spatial registration between imaging and tissue-harvesting locations. A custom metallic angled aluminum mirror ( $\sim 45$ - deg inclination) is fixed at the tip of the probe to allow tissue illumination and light detection perpendicular to the needle. The optical fibers are glued with fast curing optical adhesive (NOA81, Thorlabs) on a thin open-ended hollow metallic tube (2.11-mm internal diameter and 2.24-mm external diameter) that could fit onto the external cannula of the commercial brain biopsy needle (2.11-mm external diameter). The diameter of the resulting assembly is $2.49 \mathrm{~mm}$, including the external cannula, the hollow tube, and the fibers covered by a layer of optical glue for protection. The center-to-center distance between adjacent optical fibers is $\sim 100 \mu \mathrm{m}$.

\subsubsection{Illumination/detection system}

A fibered illumination/detection system was connected to the 18 optical fibers of the needle through SMA connectors (Fig. 2). A white light source (HL-2000 HP232, 450-1100 nm, Ocean Optics) was coupled to nine of the biopsy needle fibers using a mechanical optical switch (16-to-1 switch, MPM-2000, Ocean Optics). The illumination fibers (labeled with even numbers) alternated with the nine detection fibers on the biopsy needle (labeled with odd numbers). The latter were linked to another mechanical optical switch conducting the incoming light to a high-sensitivity spectrometer (QE65 Pro, Ocean Optics). The system was controlled using a custom Labview (National Instruments) program, automatically acquiring and storing reflectance spectra for all combinations of the illumination and detection fibers. As there were nine detection fibers and nine illumination fibers, 81 spectra in total could be acquired with the system. The acquisition time was the sum of the integration times for each spectrum plus an overall 500-ms delay associated with the motion of the mechanical components in the optical switches. In total, $13 \mathrm{~mW}$ was delivered to the distal end of the fibers for each illumination point.

\subsection{Tissue Phantom Experiments}

An experimental protocol was developed to test whether or not the new system was able to perform at the same level as the previous interstitial probe based on a stand-alone design that was not integrated onto a commercial biopsy needle. ${ }^{36}$ To this end, optical phantoms were fabricated to reproduce the geometrical and optical properties of human brain under the assumption that absorption was mostly due to $\mathrm{Hb}$, and that scattering was approximately two orders of magnitude larger than absorption. ${ }^{38,39}$ The bulk medium associated with the diffusive phantoms was composed of a solution of Intralipid ${ }^{\mathrm{TM}}$ to emulate scattering (20\%, 1:4 V/V) and blue coloring dye diluted in water for background absorption $(1: 1000 \mathrm{~V} / \mathrm{V})$. Two high-absorption carbon rods (outer diameters: $720 \mu \mathrm{m}$ for inclusion 1, $310 \mu \mathrm{m}$ for inclusion 2; see Fig. 2) were used to provide optical contrast to model blood vessels. The biopsy needle was affixed to a support, allowing its position in the bulk medium to be controlled using a three-axis translation stage (PT3, Thorlabs). Several parameters were allowed to vary in the experiments: (1) images were acquired with a varying distance between inclusion 1 and the needle, with a fixed distance between inclusion 2 and the probe, and (2) an image was acquired with the bulk medium composed only of Intralipid ${ }^{\mathrm{TM}}$ (no blue dye) as a reference for data normalization. For each dataset, the edge-to-edge

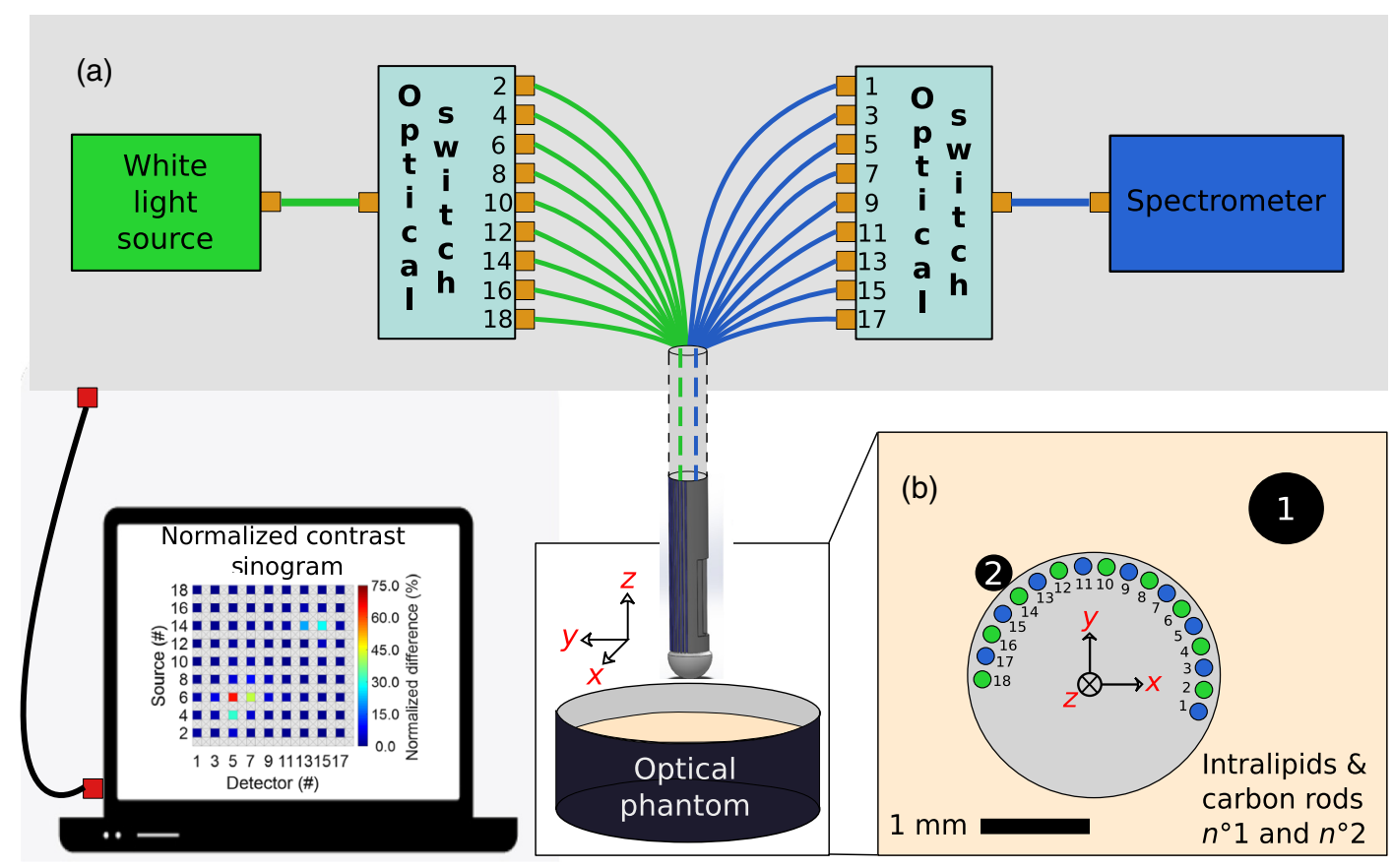

Fig. 2 (a) Schematic representations of the optical system showing the white-light source and the spectrometer connected to mechanical fiber switches allowing a wide-angle interrogation when the needle is immersed in a tissue phantom composed of multiple inclusions (black carbon rods) embedded in a scattering medium (Intralipid ${ }^{\mathrm{TM}}$ ). (b) Geometrical test configuration around the optical biopsy needle where each of the nine potentially active fiber sources are identified with an even number, whereas the nine detection sources are identified with an odd number. Those numbers are associated with the numbering scheme used for identifying the optical switch positions in (a). 
distances between inclusion 2 and the optical biopsy needle were $0,50,100,200,500,1000$, and $2000 \mu \mathrm{m}$. This resulted in the reconstruction of seven images using 81 reflectance spectra (i.e., all possible combinations of illumination/detection fibers) with an integration time for the reflectance spectra increasing from 1 to $5 \mathrm{~s}$, with larger imaging times used for larger source-detector distances. The optimal integration time was estimated for each pair of fibers to maximize usage of the dynamical range of the sensor.

\subsection{Optical Reconstruction Algorithm}

The reconstruction algorithm developed in Ref. 37 was used to image variations in optical absorption from a heterogeneous medium based on differences between data acquired in the heterogeneous medium (targeted for reconstruction) and calibration data acquired in a homogeneous medium. The technique was inspired from modeling approaches developed in diffuse optical tomography when solving the diffusion equation in the Rytov or Born approximation under the assumption that local variations in optical properties $\left(\mu_{a}, \mu_{s}^{\prime}\right)$ can be treated as perturbations. ${ }^{40,41}$ Briefly, input data for each source/detector (S/D) pair associated with a spectral band of width $\Delta \lambda$ (between wavelengths $\lambda_{i}$ and $\lambda_{f}$ ) was modeled using the following equation, where the approximation was made that the reduced scattering coefficient was constant across the imaging domain:

$I_{S / D}^{\text {homogeneous }}-I_{S / D}^{\text {heterogeneous }}=\int J\left(\vec{r}, \vec{r}_{S}, \vec{r}_{D}\right) \delta \mu_{a}(\vec{r}) \mathrm{d} \vec{r}$,

where $I_{S / D}$ is the signal intensity, $\delta \mu_{a}(\vec{r})$ is the local difference in absorption coefficient between homogeneous and heterogeneous media, and $J\left(\vec{r}, \vec{r}_{S}, \vec{r}_{D}\right)$ is the numerical sensitivity function between source $S$ and detector $D$ obtained using Monte Carlo light transport simulations. ${ }^{42,43}$ The latter function was obtained for the $S / D$ geometry associated with the fiber distribution around the needle for a homogenous background. Then, for each $S / D$ pair an estimate was computed for the variation $\delta \mu_{a}$ with respect to the homogenous background. This was achieved based on Eq. (1) under the approximation that optical property variations are constant across the imaging domain:

$\delta \mu_{a}^{S, D}=\frac{I_{S / D}^{\text {homogeneous }}-I_{S / D}^{\text {heterogeneous }}}{\int J\left(\vec{r}, \vec{r}_{S}, \vec{r}_{D}\right) \mathrm{d} \vec{r}}$.

Physically, this equation was used to assign to each $S / D$ measurement a weight quantifying its relative contribution with respect to other measurements when contributing to variations in optical properties. Finally, a reconstructed image was computed by superposing the sensitivity function of all $S / D$ pairs, each weighted by the estimate of $\delta \mu_{a}^{S, D}$.

Reconstruction process can be achieved for any spectral band by using intensities in Eqs. (1) and (2) associated with the integral of the diffuse reflectance spectra over the desired spectral domain between $\lambda_{i}$ and $\lambda_{f}$. Integrals of the whole diffusion spectra were used for the tissue phantom experiments (Sec. 2.2) but a spectral band around $575 \mathrm{~nm}(\Delta \lambda=10 \mathrm{~nm}) \mathrm{Hb}$ peak was use for the in vivo experiments (see Sec. 2.4 below) to ensure that the images reconstructed are as specific as possible to blood vessels. $^{44,45}$ The calibration data (homogenous medium) used for the swine brain experiments consisted in reflectance spectra from an Intralipid ${ }^{\mathrm{TM}}$ and blue dye phantom designed to match as closely as possible the literature values for brain tissue optical properties $\left(\mu_{a}=0.1 \mathrm{~mm}^{-1},{ }^{45,46}\right.$ and $\left.\mu_{S}^{\prime} \sim 8 \mathrm{~mm}^{-1}\right){ }^{46}$ To discriminate blood vessels from nonvascular tissue, we used Eq. (2), with $I_{S / D}^{\text {homogeneous }}$ as the intensity collected for the calibration phantom and $I_{S / D}^{\text {heterogeneous }}$ as the intensity from the interrogated medium. The weighted differences $\delta \mu_{a}^{S, D}$ were then tresholded, keeping only values at $50 \%$, or higher, of the maximum value for the whole dataset. This threshold was a free parameter of the processing that we used to maximize the correlation between the reconstructed images and the $\mathrm{x}$-ray images used for in vivo validation of the technique. A more sophisticated model, based on Monte Carlo simulations, was also developed to retrieve the optical absorption coefficient $\mu_{a}$ of the medium under the assumption of constant reduced scattering coefficient $\left(\mu_{s}^{\prime}=8 \mathrm{~mm}^{-1}\right.$ for brain tissue $\left.\mathrm{e}^{36,38,39}\right)$ Briefly, diffuse reflectance spectra were generated based on Monte Carlo simulations for a range of optical properties consistent with brain tissue. Then, using a least-squares algorithm the resulting spectral look-up table was used to associate absorption coefficient values for each wavelength of all experimental reflectance spectra. Using a curve fitting with a linear regression, each resulting $\mu_{a}$ spectrum was projected onto a basis composed of the extinction coefficients of oxygenated and deoxygenated $\mathrm{Hb}$ to retrieve their relative contributions. The resulting fitted $\mathrm{Hb}$ absorption spectra were used for image reconstruction only when $\mu_{a}>0.4 \mathrm{~mm}^{-1}$ in the $575-\mathrm{nm}$ band because this threshold was found to most consistently reconstruct vessels detected with the gold standard of x-ray imaging. However the images reconstructed with this spectral method led to the same blood vessel detection assessment when compared to Fig. 6, and as a result they were not shown for conciseness.

\subsection{In Vivo Data Acquisition Protocol}

To demonstrate the technical feasibility of using the optical brain biopsy system in an operating room, as well as its capacity for intraoperative detection of blood vessels, an in vivo experiment was designed and conducted during which it was used in a living adult swine (male, $\sim 30 \mathrm{~kg}$ ). In this experiment, the gold standard for blood vessel detection against which to evaluate the new technique was x-ray imaging. The animal ethics protocol was approved by the Comité institutionnel de protection des animaux at the Centre de recherche du Centre hospitalier de l'Université de Montréal (CRCHUM) in accordance with the Canadian Council on Animal Care guidelines. The animal was kept under general anesthesia during the entire experiment. The anesthesia was terminal.

Prior to the surgical procedure, the surgeon used two threedimensional (3-D) imaging operating modes of the X-ray system (Artis Q, Siemens, Germany). The first was computed tomography $(\mathrm{CT}, 20 \mathrm{~s}$ acquisition per scan, 1 frame every $0.5 \mathrm{deg}$, $217 \mathrm{deg}$ in total, $500-\mu \mathrm{m}$ resolution). The second imaging mode was angiography (250- $\mu \mathrm{m}$ resolution), obtained by subtracting two CT scans. For angiography, the second scan differed from the first because the contrast agent Iodixanol ${ }^{\mathrm{TM}}$ was injected $(270 \mathrm{mg} / \mathrm{mL}, 2 \mathrm{~mL} / \mathrm{s}$, and $50 \mathrm{~mL})$ to highlight the blood vessels. The contrast agent was injected through a catheter into the femoral artery $5 \mathrm{~s}$ before image acquisition to ensure that it had spread into the arteries. Based on the imaging results (3-D image of the arteries), the surgeon planned a biopsy needle insertion trajectory to minimize the risks of damaging critical vessels. Although angiography had superior spatial resolution, CT scan images were shown to be more sensitive to vessels and thus were used to provide a postoperative 
reference in order to preliminarily assess the ability of the optical biopsy system to detect the presence of blood vessels.

During insertion of the biopsy needle, the fluoroscopy modality of the x-ray imaging system was used as a guide. Two two-dimensional (2-D) fluoroshots were saved at several points along the trajectory and combined to retrieve the 3-D location of the biopsy tool. At each imaging position, 30 reflectance spectra were acquired to reconstruct an image instead of the 81 used previously in the in vitro experiments. This constraint was imposed by the need to ensure that the full procedure was kept under $60 \mathrm{~min}$ as stipulated in the animal protocol. This resulted in an integration time of $55 \mathrm{~s}$ per image for each of the nine different depth locations along the trajectory of the optical biopsy needle. The spatial insertion step between two locations along the trajectory of the needle was $\sim 3 \mathrm{~mm}$. After the ninth acquisition, the target was reached covering a total insertion length of $23 \mathrm{~mm}$ from the initial depth. The optical biopsy needle was then removed. Each spatial position where optical data were acquired was then retrieved from the 2-D fluoroshots and merged with a CT scan. This resulted in a 3-D image map of blood vessels in the brain over which the trajectory of the needle was superposed. Image merging was done using the Medical Imaging Interaction Toolkit (MITK) ${ }^{47}$ to provide reference data for blood vessel localization. Quantitative estimation of the registration error associated with disruption of the vessels is left for future work and remains here evaluated to $\sim 1$ to $2 \mathrm{~mm}$.

\section{Results}

\subsection{Tissue Phantom Experiment}

The aim of the phantom experiments is to reproduce a subset of results obtained in Refs. 36 and 37 with a different probe design in order to demonstrate that the current system can also localize highly absorbing inclusions. For succinctness, images are presented only for a subset of the phantoms that are studied. Figure 3 shows reconstruction results of 4 out of the 28 phantoms that are imaged in this work. The figure shows images of

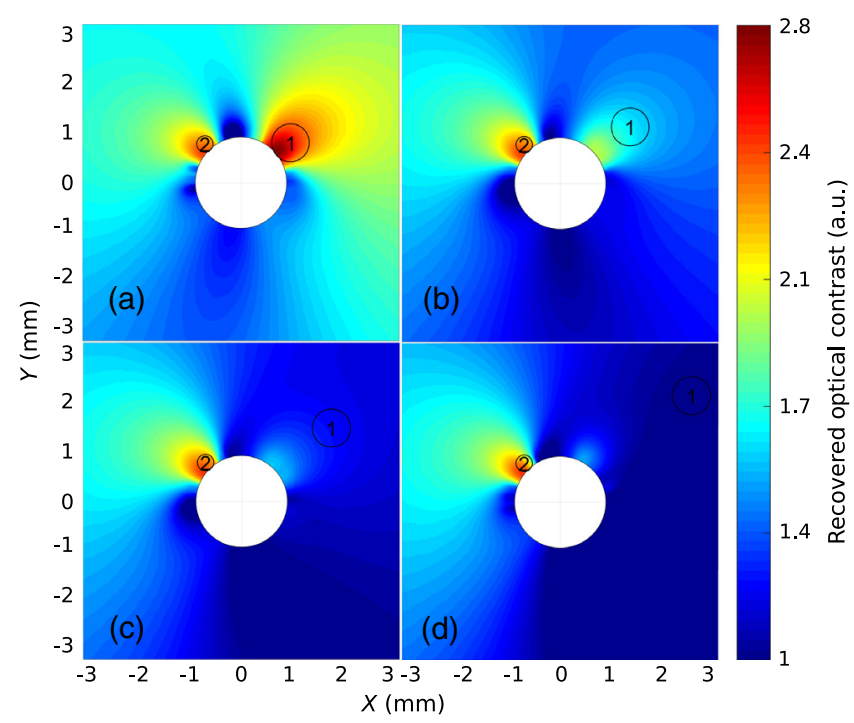

Fig. 3 Reconstructed diffuse optical images of the carbon rods immersed in a diffusive medium. The distances (edge to edge) between the optical biopsy needle and inclusion 1 are (a) $0 \mathrm{~mm}$, (b) $0.5 \mathrm{~mm}$, (c) $1 \mathrm{~mm}$, and (d) $2 \mathrm{~mm}$. The other carbon rod (inclusion 2) remains at the same position in all experiments. carbon rods immersed in a diffusive medium with optical properties $\mu_{a}=0.01 \mathrm{~mm}^{-1}$ and $\mu_{s}^{\prime}=8 \mathrm{~mm}^{-1}$, with edge-to-edge distances between the optical biopsy needle and inclusion 1: (a) $0 \mathrm{~mm}$, (b) $0.5 \mathrm{~mm}$, (c) $1 \mathrm{~mm}$, and (d) $2 \mathrm{~mm}$. The other carbon rod (inclusion 2) remains at the same position in all four phantoms. For each image, the location of the carbon rods is indicated with black circles, whereas the color scale values (contrast) can be interpreted as being proportional to the probability that an absorber is present at the location of a given pixel. The cross section of the biopsy needle is represented with the white circle in the center of each image. The results in Fig. 3 demonstrate a match between the estimated angular location of the inclusion around the probe and its actual position for each of the four datasets. Furthermore, the decrease of the optical contrast when inclusion 1 is moved away from the edge of the probe shows the radial sensitivity of the system to be on the order of $2 \mathrm{~mm}$ from the edge of the probe. This is consistent with the radial sensitivity found in Refs. 36 and 37, and this finding is also supported by the reconstructed images (not shown for conciseness) of the other 24 phantoms with different background optical properties. Here depth sensitivity to inclusion 1 has been assessed based on whether any contrast could be observed in the radial direction from the needle to the inclusion. For example, although small for larger distances between inclusion 1 and the needle, contrast could still be observed up to an edgeto-edge separation of $2 \mathrm{~mm}$ despite the presence of a large contrast absorber next to the needle (inclusion 2). Each measured spectrum satisfies the requirement that its signal-to-noise ratio (SNR) is superior to 5 , using the formula SNR $=\sqrt{I}$, where $I$ is the number of photon counts in the 450- to $950-\mathrm{nm}$ spectral band. For this experiment the SNR value estimated for each reflectance spectra is between 7 and 83 , with an average value of 22 and a standard deviation of 18 .

\subsection{In Vivo Experiment}

To evaluate the potential of the system to detect blood vessels in vivo, the 3-D CT image map is analyzed for use as the gold standard for vessels' detection. Figure 4(a) shows a large field-ofview image of the CT volume, whereas Fig. 4(b) shows an enlarged view around the vicinity of the probe. In that enlarged view, the needle is shown in white, whereas the system holding

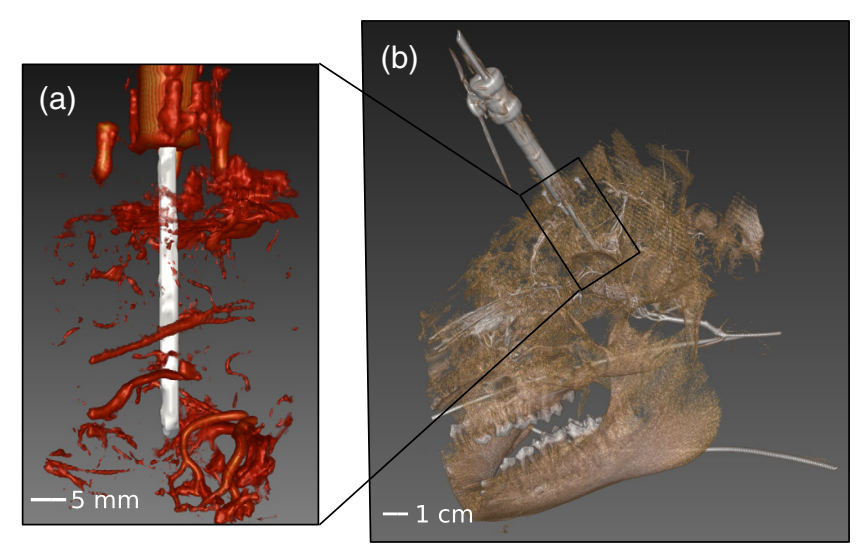

Fig. 4 CT scan used for locating blood vessels in the vicinity of the biopsy needle: (a) representation of blood vessels (red) surrounding the optical biopsy needle (gray tubular object) and (b) 3-D reconstruction of the surgical field in semitransparency showing the needle (in gray), the swine skull (in brown), and the blood vessels (in white). 
(a)

(b)

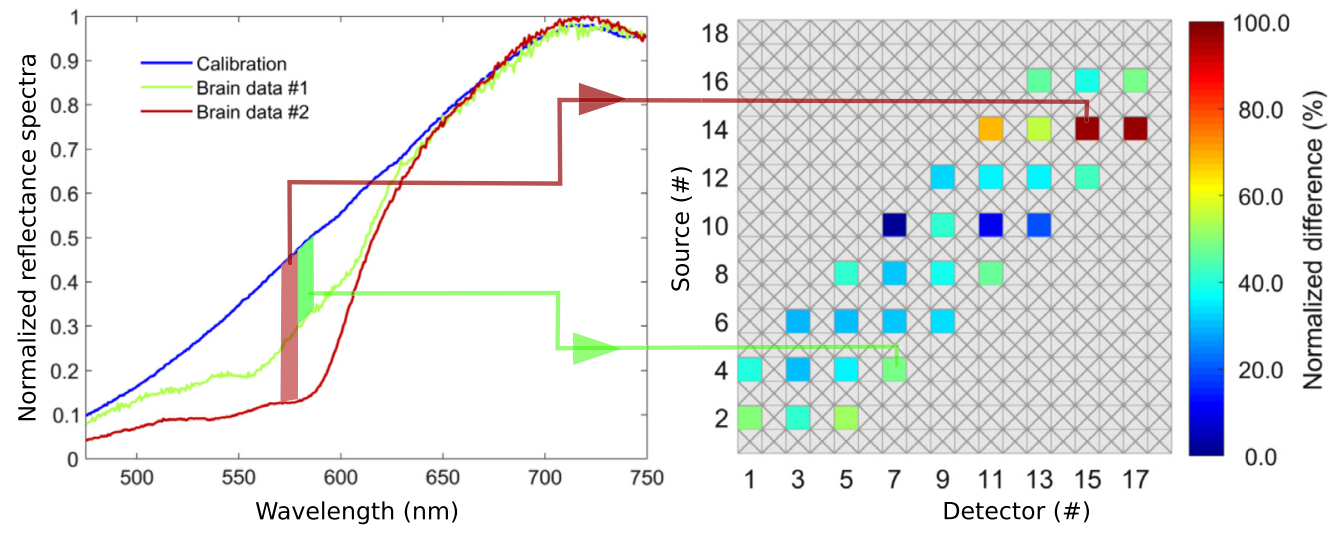

Fig. 5 (a) Representative in vivo diffuse reflectance spectra and (b) optical sinogram associated with an interstitial measurement in swine brain.

it (made of a titanium tube and three screws) and the detected vessels are all shown in red. The large field-of-view image in the figure shows the swine skull along with the holding system and the needle. The reconstruction of the blood vessel network inside the brain provides the information necessary to estimate the location and the size of the vessels in the close vicinity of the needle. The precision for the vessels' localization is limited by the error registration of the probe and the 3-D CT image map roughly estimated to $1 \mathrm{~mm}$.

Figure 5 shows two representative diffuse reflection spectra out of the 30 that are acquired per biopsy needle position, along with the calibration spectrum associated with a homogenous medium. Each spectrum in the figure shows characteristic features of the $\mathrm{Hb}$ absorption signature, including an increased absorption around $575 \mathrm{~nm}$. The figure also shows the optical sinogram for one needle position, which graphically represents intensities associated with all 30 reflectance spectra with the detector number on the vertical axis and the source number on the horizontal axis. The intensity values in the sinogram are associated with the difference between the in vivo data and the calibration data (homogenous medium) at $575 \mathrm{~nm}$. The sinogram data are used directly as input in Eq. (2) for image reconstruction. For this experiment, each measured spectrum used for image reconstruction satisfies the requirement that its SNR is superior to 5 in the spectral range from 570 to $580 \mathrm{~nm}$. The SNR value estimated for each reflectance spectra is then between 5 and 107, with an average value of 38 and a standard deviation of 22 .

Figure 6 shows a 3-D representation of the blood vessel distribution detected in swine brain using CT and diffuse optical imaging. For comparison purposes, each of the nine diffuse optical 2-D images [Fig. 6(a)] are juxtaposed with their corresponding 2-D CT slice from the full 3-D volume to assess the potential of the optical needle system to detect vessels. Importantly, all diffuse reflectance spectra are inspected and they show characteristic features of $\mathrm{Hb}$, confirming that the optical contrast is not provided by the $\mathrm{X}$-ray contrast agent itself, which had cleared the vessels' network at the moment when the diffuse reflectance images are acquired. The $\mathrm{Hb}$ features themselves are the high optical absorption in the near 570 to $580 \mathrm{~nm}$ region and the low absorption elsewhere in the spectral range of the imaging system. An experienced radiologist (GS) analyzed the CT images to discriminate vessels from image noise and was able to confirm that the contrast observed in Fig. 6(b) is (a)
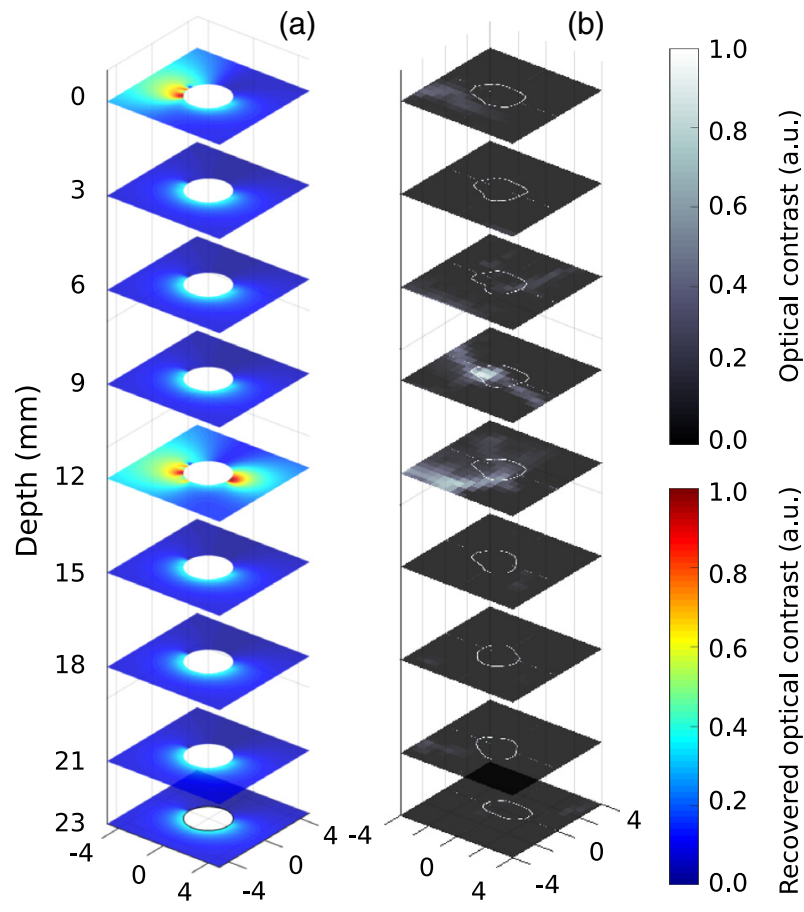

Fig. 6 Comparison of spatially coincident images: (a) diffuse optical reconstructions and (b) CT scan ( $x$-axis and $y$-axis units are in millimeters).

associated with blood vessels. For the slices at depth 3, 6, $15,18,21$, and $23 \mathrm{~mm}$, both the diffuse optical image and the CT slice are consistent with an absence of blood vessels. There is also a match for depths 0 and $12 \mathrm{~mm}$ where contrast is observed in both the diffuse optical and the CT images. However, the slice at depth $9 \mathrm{~mm}$ can be considered as a false negative for diffuse optical image as the CT scan image shows blood vessel contrast not detected with the optical imaging system.

\section{Discussion}

The preliminary in vivo results presented in this work suggest that a diffuse optical technique could detect blood vessels using $\mathrm{Hb}$ absorption as a surrogate for blood vessels, as demonstrated by comparison with contrast $\mathrm{CT}$ used here as the gold standard 
for vessels' detection. In its current form, we believe the system presents features making in-human clinical translation eventually possible. For example, the technique could be used intraoperatively since the number of steps needed to manipulate the needle inside the brain is limited and does not require the use of any contrast agent.

Although the current optical reconstruction algorithm is capable of detecting high-absorbing inclusions in a diffusive medium up to $\sim 2 \mathrm{~mm}$ from the needle core, it remains limited in terms of depth specificity, making it almost impossible to differentiate a large inclusion away from the edge of the needle and a smaller one in a closest range. As proposed in Ref. 36, this limitation could be overcome by using a more sophisticated reconstruction algorithm akin to diffuse optical tomography, albeit adapted to an outside-viewing interstitial imaging geometry. Furthermore, the method used to merge CT and diffuse optical images is inherently limited and prone to errors. This is because the CT image used as the gold standard is acquired prior to inserting the biopsy needle into the brain. As a result, needle insertion may have incurred shifts in vessels' positions introducing a spatial mismatch between the high-contrast locations predicted by both methods. For example, the false-negative vessel detection for diffuse optical imaging in Fig. 6(b) might simply be a consequence of the vessels seen in the CT slice having moved in front of the dead angle of the optical detection system. To solve this problem, we consider acquiring the CT scan only once the optical biopsy needle is inserted instead of prior to. However, this is made impossible due to artifacts caused by the metal in the needle and the limited imaging time allowed by the ethics protocol.

Several aspects are in need of improvement prior to first inhuman testing. Most importantly, the diffuse optical imaging time per needle position needs to be reduced to $<1 \mathrm{~s}$ in order to minimize the disruption in the surgical workflow; here, the total in vivo imaging time is $55 \mathrm{~s}$. However, this is achieved by using only a limited number of source-detector fiber pairs, namely only those for which the angular separation did not exceed $23 \mathrm{deg}$. Although this reduced the integration time significantly (from $\sim 7 \mathrm{~min}$ to $55 \mathrm{~s}$ ), using a limited number of fibers also reduced the sensitivity of the system, potentially explaining the false negative diffuse optical image in Fig. 4 for the slice at depth $9 \mathrm{~mm}$. Ensuring that all fibers are used for a total integration in $<1 \mathrm{~s}$ can be achieved through a system redesign where instead of using a white-light lamp and a spectrometer, a simple laser diode centered at $575 \mathrm{~nm}$ is used in conjunction with an array of single-point detectors (e.g., photomultiplier tube or avalanche photodiode), each dedicated to one detection fiber. Light delivery to tissue in the current prototype is also highly inefficient because of suboptimal fiber-optic couplings leading to light doses delivered to tissue at least two orders of magnitude smaller than the maximum permissible exposure (MPE) limits for skin. As a result, integration time could be reduced dramatically by simply improving the optical design and by using a higher power light source.

Future work will also involve the conduct of a clinical study and the development of a validation protocol of the new technique based on preoperative contrast-enhanced magnetic resonance imaging (MRI) rather than contrast CT because the latter is not a neuroimaging standard in oncology. In particular, the protocol will involve tracking the needle position in real time using a neuronavigation system allowing preoperative MRIs to be spatially registered with each position where an optical measurement and a biopsy sample are collected. The presence of tissue damage associated with tissue harvesting will be assessed based on histopathology analyses and the presence of microvessels will be determined using immunohistochemistry using appropriate markers. Although the current approach can potentially improve the safety of brain biopsy procedures, an instrument synergistically integrating a biomolecular detection modality (e.g., Raman spectroscopy ${ }^{48-51}$ ) with the diffuse optical approach could increase the diagnostic yield of the procedure to reduce instances of repeat procedures and limit the number of biopsy samples per procedure.

\section{Conclusion}

We have designed, fabricated, and validated a diffuse optical imaging system directly integrated on a commercial biopsy needle with the objective to detect blood vessels interstitially in the brain. The capabilities of the instrument were first demonstrated through an in vitro experiment using tissue phantoms to show that it can detect high absorbing inclusions within a diffusive medium. In vivo results in one animal were also presented to demonstrate feasibility and practicality of the technique in a clinical environment as well as the application of a protocol using CT imaging to validate the blood vessel detection capabilities of the optical system.

\section{Disclosures}

We do not declare any conflict of interest concerning this study.

\section{Acknowledgments}

This work is supported by the Discovery Grant program from Natural Sciences and Engineering Research Council of Canada (NSERC), the Collaborative Health Research Program (CIHR and NSERC), and the company Medtronic, which provided support with the commercial brain biopsy needles. We would also like to thank Optech for its help with fiber alignment and connections. Finally, we thank the personnel of the Animal Core Facility of the CRCHUM for providing the animal care and anesthesia and the CRCHUM Imagery Core Facility for help and expertise in imagery procedures.

\section{References}

1. C. Nimsky et al., "Volumetric assessment of glioma removal by intraoperative high-field magnetic resonance imaging," Neurosurgery 55, 358-370; discussion pp. 370-371 (2004).

2. E. B. Claus et al., "Survival rates in patients with low-grade glioma after intraoperative magnetic resonance image guidance," Cancer 103, 1227-1233 (2005).

3. M. Lacroix et al., "A multivariate analysis of 416 patients with glioblastoma multiforme: prognosis, extent of resection, and survival," J. Neurosurg. 95, 190-198 (2001).

4. E. R. Laws et al., "Surgical management of intracranial gliomas-does radical resection improve outcome?" Acta Neurochir. Suppl. 85, 47-53 (2003).

5. J. C. Buckner, "Factors influencing survival in high-grade gliomas," Semin. Oncol. 30, 10-14 (2003).

6. G. E. Keles, K. R. Lamborn, and M. S. Berger, "Low-grade hemispheric gliomas in adults: a critical review of extent of resection as a factor influencing outcome," J. Neurosurg. 95, 735-745 (2001).

7. S. A. Yeh et al., "Treatment outcomes and prognostic factors in patients with supratentorial low-grade gliomas," Br. J. Radiol. 78, 230-235 (2005).

8. J. P. Schneider et al., "Gross-total surgery of supratentorial low-grade gliomas under intraoperative MR guidance," Am. J. Neuroradiol. 22, 89-98 (2001). 
9. R. Dammers et al., "Safety and efficacy of frameless and frame-based intracranial biopsy techniques," Acta Neurochir. (Wien) 150, 23-29 (2008).

10. M. D. Krieger et al., "Role of stereotactic biopsy in the diagnosis and management of brain tumors," Semin. Surg. Oncol. 14, 13-25 (1998).

11. A. V. Kulkarni et al., "Incidence of silent hemorrhage and delayed deterioration after stereotactic brain biopsy," J. Neurosurg. 89, 31-35 (1998).

12. M. Field et al., "Comprehensive assessment of hemorrhage risks and outcomes after stereotactic brain biopsy," J. Neurosurg. 94, 545-551 (2001).

13. R. Grossman et al., "Haemorrhagic complications and the incidence of asymptomatic bleeding associated with stereotactic brain biopsies," Acta Neurochir. (Wien) 147, 627-631; discussion 631 (2005).

14. D. Kondziolka, A. D. Firlik, and L. D. Lunsford, "Complications of stereotactic brain surgery," Neurol. Clin. 16, 35-54 (1998).

15. W. A. Hall, "The safety and efficacy of stereotactic biopsy for intracranial lesions," Cancer 82, 1749-1755 (1998).

16. L. D. Lunsford et al., "Establishing a benchmark for complications using frame-based stereotactic surgery," Stereotact. Funct. Neurosurg. 86, 278-287 (2008).

17. J. Tilgner et al., "Validation of intraoperative diagnoses using smear preparations from stereotactic brain biopsies: intraoperative versus final diagnosis-influence of clinical factors," Neurosurgery 56, 257-265; discussion 257-265 (2005).

18. D. U. Bartsch et al., "3-D reconstruction of blood vessels in the ocular fundus from confocal scanning laser ophthalmoscope ICG angiography," in Proc. Int. Conf. Image Process., IEEE, Vol. 3, pp. 687-690 (1996).

19. L. Boni et al., "Clinical applications of indocyanine green (ICG) enhanced fluorescence in laparoscopic surgery," Surg. Endosc. 29(7), 2046-2055 (2015).

20. A. Rühm et al., "ICG-assisted blood vessel detection during stereotactic neurosurgery: simulation study on excitation power limitations due to thermal effects in human brain tissue," Photodiagn. Photodyn. Ther. 11(3), 307-318 (2014).

21. M. Hassan et al., "Near infrared fluorescence imaging with ICG in TECAB surgery using the da Vinci Si surgical system in a canine model," J. Cardiac Surg. 27(2), 158-162 (2012).

22. Y. Rino et al., "Visualization of blood supply route to the reconstructed stomach by indocyanine green fluorescence imaging during esophagectomy," BMC Med. Imaging 14(1), 18 (2014).

23. J. de Moura et al., "Enhanced visualization of the retinal vasculature using depth information in OCT," Med. Biol. Eng. Comput. 55(12), 2209-2225 (2017).

24. S. Yang et al., "OCT imaging detection of brain blood vessels in mouse, based on semiconducting polymer nanoparticles," Analyst 142(23), 4503-4510 (2017).

25. P. Gong et al., "Optical coherence tomography for longitudinal monitoring of vasculature in scars treated with laser fractionation," J. Biophotonics 9(6), 626-636 (2016).

26. D. Lorenser et al., "Dual-modality needle probe for combined fluorescence imaging and three-dimensional optical coherence tomography," Opt. Lett. 38(3), 266-268 (2013).

27. S. Onoda et al., "Preoperative identification of perforator vessels by combining MDCT, Doppler flowmetry, and ICG fluorescent angiography," Microsurgery 33(4), 265-269 (2013).

28. R. A. Leitgeb et al., "Real-time assessment of retinal blood flow with ultrafast acquisition by color Doppler Fourier domain optical coherence tomography," Opt. Express 11(23), 3116-3121 (2003).

29. H. Ramakonar et al., "Intraoperative detection of blood vessels with an imaging needle during neurosurgery in humans," Sci. Adv. 4(12), eaav4992 (2018).
30. C. P. Liang et al., "Coherence-gated Doppler: a fiber sensor for precise localization of blood flow," Biomed. Opt. Express 4(5), 760-771 (2013).

31. V. Rajan et al., "Review of methodological developments in laser Doppler flowmetry," Lasers Med. Sci. 24(2), 269-283 (2009).

32. K. Wårdell et al., "High-resolution laser Doppler measurements of microcirculation in the deep brain structures: a method for potential vessel tracking," Stereotactic Funct. Neurosurg. 94(1), 1-9 (2016).

33. V. Virdyawan et al., "Laser Doppler sensing for blood vessel detection with a biologically inspired steerable needle," Bioinspiration Biomimetics 13(2), 026009 (2018).

34. M. A. Davis et al., "Sensitivity of laser speckle contrast imaging to flow perturbations in the cortex," Biomed. Opt. Express 7(3), 759-775 (2016).

35. K. Basak, M. Manjunatha, and P. K. Dutta, "Review of laser specklebased analysis in medical imaging," Med. Biol. Eng. Comput. 50(6), 547-558 (2012).

36. J. Pichette et al., "Sensitivity analysis aimed at blood vessels detection using interstitial optical tomography during brain needle biopsy procedures," Biomed. Opt. Express 6(11), 4238-4254 (2015).

37. A. Goyette et al., "Sub-diffuse interstitial optical tomography to improve the safety of brain needle biopsies: A proof-of-concept study," Opt. Lett. 40(2), 170-173 (2015).

38. P. Van Der Zee, M. Essenpreis, and D. T. Delpy, "Optical properties of brain tissue," Proc. SPIE 1888, 454-465 (1993).

39. A. N. Yaroslavsky et al., "Optical properties of selected native and coagulated human brain tissues in vitro in the visible and near infrared spectral range," Phys. Med. Biol. 47(12), 2059-2073 (2002).

40. S. R. Arridge, "Optical tomography in medical imaging," Inverse Probl. 15(2), R41 (1999).

41. D. A. Boas et al., "Three dimensional Monte Carlo code for photon migration through complex heterogeneous media including the adult human head," Opt. Express 10(3), 159-170 (2002).

42. Q. Fang, "Mesh-based Monte Carlo method using fast ray-tracing in Plucker coordinate," Biomed. Opt. Express 1(1), 165-175 (2010).

43. Q. Fang and D. Boas, "Tetrahedral mesh generation from volumetric binary and gray-scale images," in Proc. IEEE Int. Symp. Biomed. Imaging, pp. 1142-1145 (2009).

44. S. Prahl, "Optical absorption of hemoglobin," Oregon Medical Laser Center, http://omlc.ogi.edu/spectra/hemoglobin/index.html.

45. M. Mastanduno, "Combined MRI and near-infrared spectroscopy for increased specificity of breast cancer imaging," Dartmouth College, https://cpb-us-e1.wpmucdn.com/sites.dartmouth.edu/dist/1/627/files/ 2014/01/Mastanduno_thesis2.pdf (2013).

46. P. Di Ninni, F. Martelli, and G. Zaccanti, "Intralipid: towards a diffusive reference standard for optical tissue phantoms," Phys. Med. Biol. 56(2), N21D (2010).

47. I. Wolf et al., "The medical imaging interaction toolkit," Med. Image Anal. 9(6), 594-604 (2005).

48. M. Jermyn et al., "Intraoperative brain cancer detection with Raman spectroscopy in humans," Sci. Transl. Med. 7(274), 274ra19 (2015).

49. M. Jermyn et al., "Highly accurate detection of cancer in situ with intraoperative, label-free, multimodal optical spectroscopy," Cancer Res. 19, 1-10 (2017).

50. J. Desroches et al., "A new method using Raman spectroscopy for in vivo targeted brain cancer tissue biopsy," Sci. Rep. 8(1), 1792 (2018).

51. J. Desroches et al., "Development and first in-human use of a Raman spectroscopy guidance system integrated with a brain biopsy needle," J. Biophotonics 12(3), (2019).

Biographies of the authors are not available. 